\title{
Recent Advances in Symmetry Groups and Conservation Laws for Partial Differential Equations and Applications
}

\author{
Maria Gandarias, ${ }^{1}$ Mariano Torrisi, ${ }^{2}$ Maria Bruzón, ${ }^{1}$ \\ Rita Tracinà, ${ }^{2}$ and Chaudry Masood Khalique ${ }^{3}$ \\ ${ }^{1}$ Departamento de Matematicas, Universidad de Cádiz, Puerto Real, 11510 Cádiz, Spain \\ ${ }^{2}$ Dipartimento di Matematica e Informatica, Università di Catania, 95125 Catania, Italy \\ ${ }^{3}$ Department of Mathematical Sciences, International Institute for Symmetry Analysis and Mathematical Modelling, \\ North-West University, Mafikeng Campus, Mmabatho 2735, South Africa
}

Correspondence should be addressed to Maria Gandarias; marialuz.gandarias@uca.es

Received 25 August 2014; Accepted 25 August 2014; Published 22 December 2014

Copyright (C) 2014 Maria Gandarias et al. This is an open access article distributed under the Creative Commons Attribution License, which permits unrestricted use, distribution, and reproduction in any medium, provided the original work is properly cited.

Differential equations govern many natural phenomena and play an important role in the progress of engineering and technology. Essentially a lot of the fundamental equations are nonlinear and in general such nonlinear equations are often very difficult to solve explicitly. Symmetry group techniques provide methods to obtain solutions of these equations. These methods have several applications in the studies of partial differential equation. They are also useful in the search for conservation laws which arises in many fields of the applied sciences. Recent studies have shown that infinitely many nonlocal symmetries of various integrable models are related to their Lax pairs. Moreover symmetry method is one of the most powerful tools that give new integrable models from known ones. Integrable models have played an important role in applied sciences and are one of the central topics in soliton theory. In order to know if a system is integrable, it is very important to study Lax pairs of the system.

A symmetry can be considered as an equivalence transformation which leaves invariant not only the differential structure of equation but also the form of the arbitrary elements. This fact made Ovsiannikov search for equivalence transformations in a systematic way by using an algorithm based on the extension of the Lie infinitesimal criterion.

When an equation contains an arbitrary function, it reflects the individual characteristic of the phenomena belonging to a large class. In this sense, the knowledge of equivalence transformations can provide us with certain relations between the solutions of different phenomena of the same class.

Nowadays several branches of the theoretical and applied sciences such as mathematics, physics, biology, economy, and finance rely on processes which are usually modeled by nonlinear differential equations. Often it is difficult to obtain reductions and exact solutions for these models. Our aim is to highlight applications of symmetry methods to nonlinear models in physics, engineering, and the applied science as well as to show recent theoretical developments in symmetry groups and geometric methods.

The authors of this special issue had been invited to submit original research articles as well as review articles in the following topics: advanced researches and theoretical analyses in group transformations and differential equations; Noether symmetries, applications, and conservation laws; numerical algorithms concerning the symmetry groups for partial differential equations; new and direct methods to obtain exact explicit solutions for differential equations; applications: novel applications in sciences, including engineering, physics, biology, and finance; and reviews: lucid surveys and review articles dealing with modern and classical topics.

However, we received 20 papers in these research fields. After a rigorous reviewing process, twelve articles were finally 
accepted for publication. These articles contain some new and innovative techniques and ideas that may stimulate further researches in several branches of theory and applications of the transformation groups.

\title{
Acknowledgments
}

We would like to thank all the authors who sent their works and all the referees for the time spent in reviewing the papers. Their contributions and their efforts have been very important for the publication of this special issue.

\author{
Maria Gandarias \\ Mariano Torrisi \\ Maria Bruzón \\ Rita Tracinà
} Chaudry Masood Khalique 


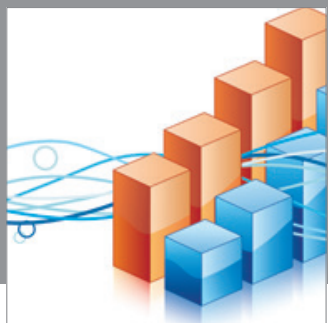

Advances in

Operations Research

mansans

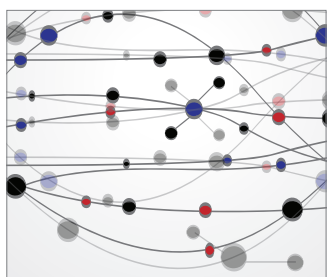

The Scientific World Journal
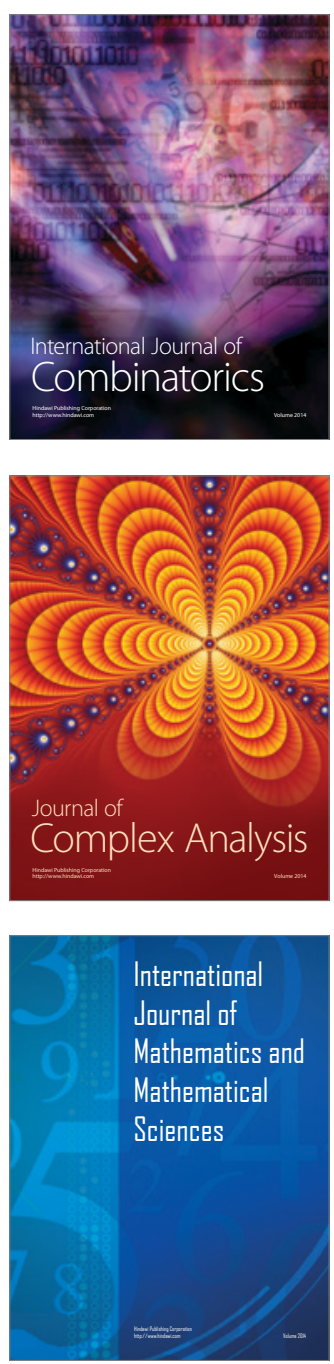
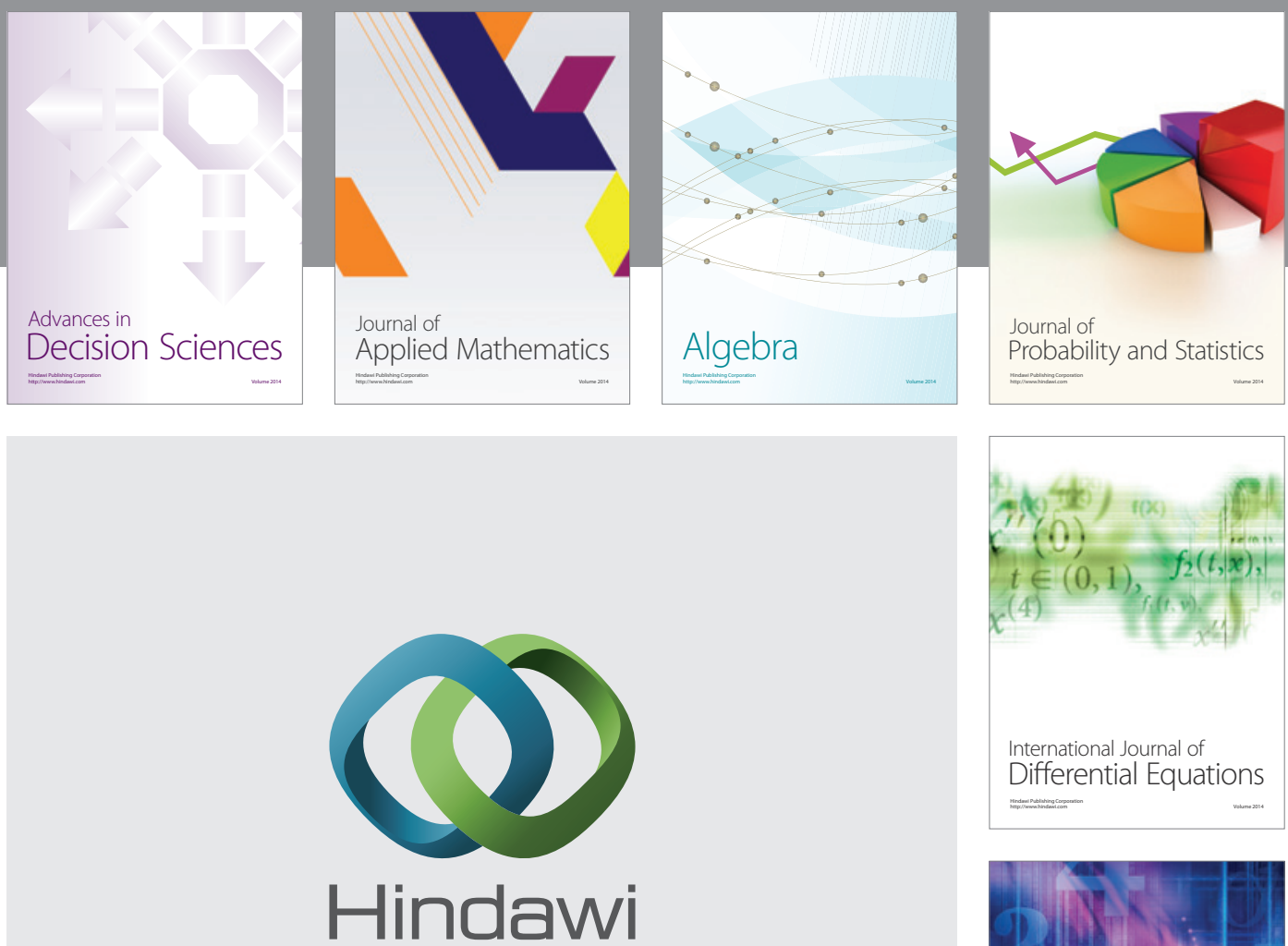

Submit your manuscripts at http://www.hindawi.com
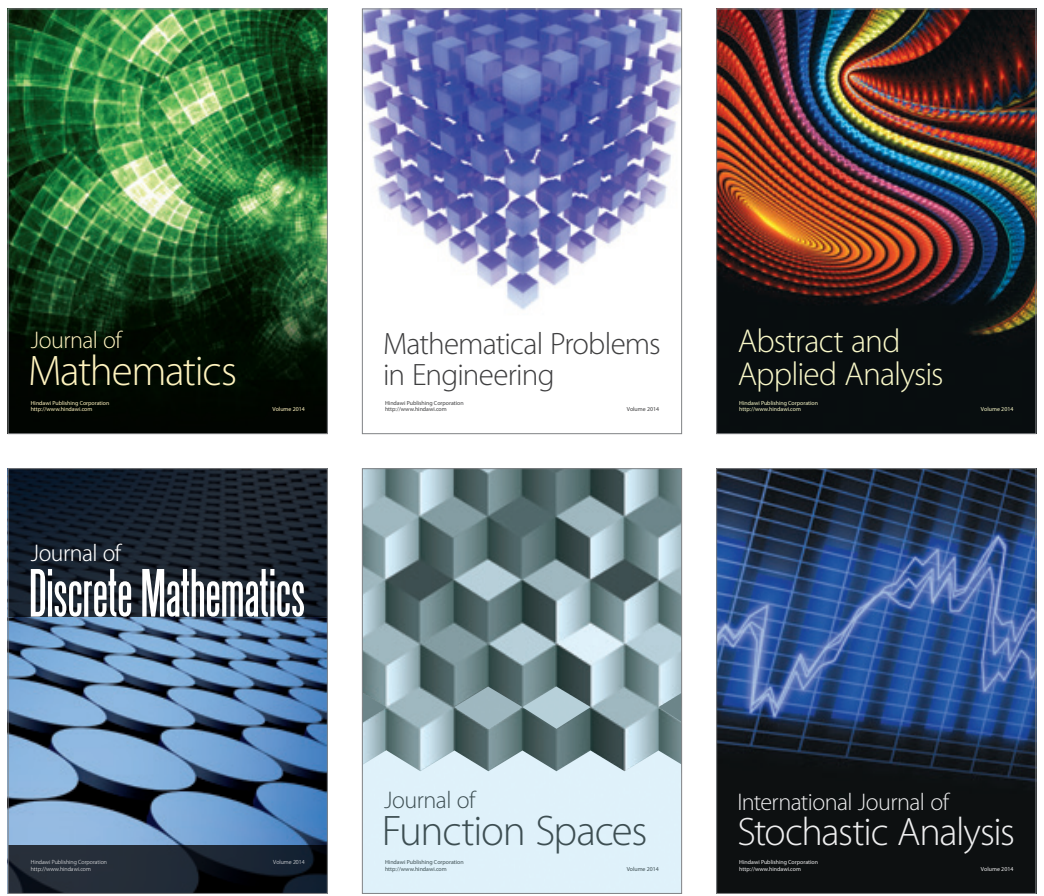

Journal of

Function Spaces

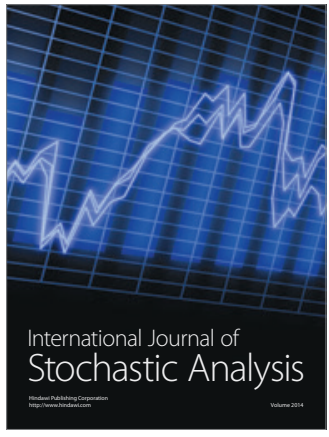

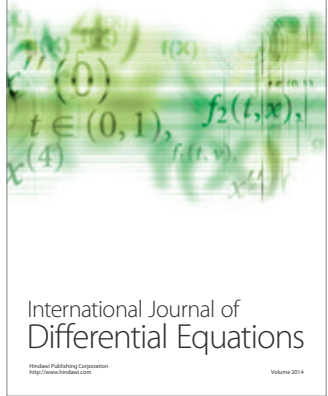
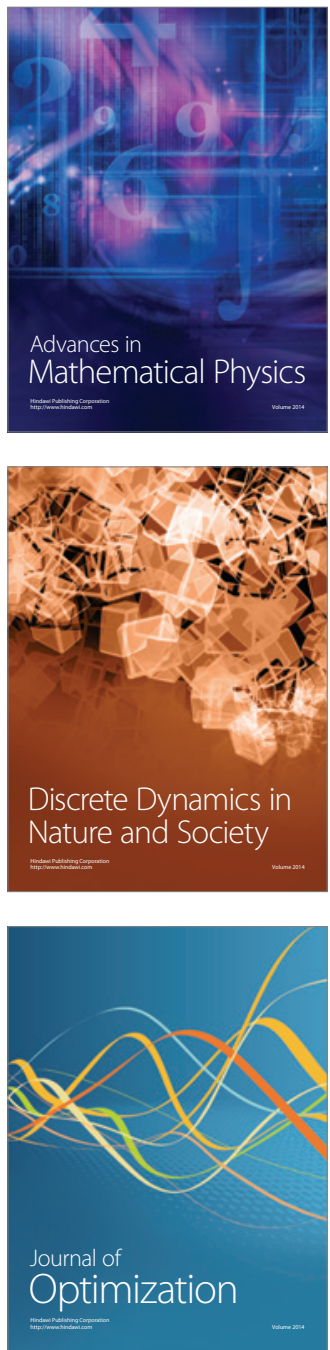